\title{
Comparación entre sistemas electrocerámicos de desplazamiento mecánico. Motores y actuadores piezoeléctricos
}

\author{
D. MESONERO-ROMANOS ${ }^{1}$, J.F. FERNÁNDEZ ${ }^{2}$, M. VILLEGAS ${ }^{2}$, R. CERES ${ }^{1}$, E. ROCON, J.L. PONS ${ }^{1}$ \\ 'Instituto de Automática Industrial, CSIC, Ctra. Campo Real, km. 0,200, 28500 Arganda del Rey, Madrid \\ ${ }^{2}$ Instituto de Cerámica y Vidrio, CSIC, Camino de Valdelatas s/n, 28049 Madrid
}

\begin{abstract}
En general, al clasificar los actuadores piezoeléctricos, se puede hablar de motores de respuesta resonante o forzada. Dentro de los motores resonantes, cabe destacar los motores, tanto lineales como rotativos, de onda viajera. En estos dispositivos existe una serie de hechos diferenciadores con respecto a cualquier otro tipo de dispositivos piezoeléctricos que los hacen relativamente más complejos: problemas de contacto intermitente entre sólidos, oscilación sincronizada en ejes perpendiculares, excitación en régimen próximo a la resonancia. Todos estos aspectos han limitado la aplicación de este tipo de actuadores, si bien sus características de accionamiento silencioso, con elevado par de salida y su construcción compacta los hacen adecuados para numerosas aplicaciones. Por otro lado, los actuadores piezoeléctricos con excitación forzada, por el hecho de que no existe amplificación de su movimiento en resonancia, se han empleado en aquellas aplicaciones en las que se requiere posicionamiento ultrapreciso con niveles de esfuerzo medios. Entre estos dispositivos cabe resaltar los actuadores lineales multicapa y cíclicos. El presente trabajo pretende recoger, tras analizar las principales características de los actuadores resonantes y forzados, las aplicaciones que es previsible se desarrollarán en los próximos años. Con el fin de resaltar los pasos necesarios hasta alcanzar su aplicación comercial, analizaremos las líneas de trabajo que en el pasado y actualmente se han identificado como más relevantes y se indicará su posible evolución en el futuro.
\end{abstract}

Palabras clave: motores piezoeléctricos, actuadores electrocerámicos, motores resonantes, FEM

\section{Comparison between mechanical displacement electroceramic systems: Piezoelectric motors and actuators}

Piezoelectric motors can be classified as resonant or forced response motors. As to resonant motors, travelling wave motors, either rotary or lineal must be highlighted. These devices have several features that make them different as compared to any other type of piezoelectric devices. In general, they are comparatively more complex since they include intermittent contact problems between solids, synchronized oscillations in perpendicular axes, and excitation close to the resonance. All these features have limited the application of this kind of actuators, even though some functional characteristics, as noiseless operation, high torque range and their compact construction have made them suitable for several applications. On the other hand, piezoelectric actuators with forced excitation, due to their non-existence movement amplification in the resonance, have been used in applications where an ultra-accurate positioning is required with intermediate levels of force. Lineal multilayer and cyclic actuators must be identified as classical forced actuators.

The aim of this paper is to summarize, analyzing the main features of the resonant and forced actuators, the applications where this type of motors will be used in the future. With the intention of remark the steps needed to achieve the commercial application, past and nowadays most important researching lines will be studied and a possible evolution in the future will be pointed out.

Keywords: Piezoelectric motors, electro-ceramic actuators, resonant motors, EMF.

\section{INTRODUCCIÓN}

El concepto general de todo motor de estado sólido es el generar un movimiento mecánico macroscópico a través de la amplificación y repetición de micro-deformaciones de materiales activos. En el caso de los motores electrocerámicos la conversión de energía eléctrica a energía mecánica (ya sea de rotación o translación) se realiza a través del efecto piezoeléctrico inverso de las electrocerámicas que llevan incorporadas estos motores.

Los motores electrocerámicos pueden ser clasificados de diversas formas. Teniendo en cuenta el tipo de excitación nos encontramos con que los motores pueden ser resonantes o forzados. La proximidad de la excitación del motor a una de las frecuencias de resonancia implica que se aprovecha la amplificación mecánica que se produce en estas frecuencias, por lo que los motores de excitación forzada obtendrán desplazamientos relativamente más bajos que los resonantes, viéndose sin embargo eximidos de los problemas que supone excitar en resonancia un motor.

Además de las diferencias existentes en cuanto a la amplitud de los desplazamientos obtenidos en los actuadores forzados o resonantes cabe destacar la diferencia existente en el diseño de la electrónica de control y potencia de cada uno de ellos. Mientras que en el caso de los forzados la electrónica no presenta inconvenientes reseñables, la de los resonantes lleva consigo el problema de tener que excitar a la frecuencia de resonancia. Para que este funcionamiento sea lo más eficiente posible, es necesario diseñar el circuito electrónico para que su funcionamiento óptimo sea a la frecuencia de resonancia del motor y así tener menos pérdidas. Existen diversos métodos para la generación y seguimiento de las señales de excitación en motores resonantes generalmente basados en PLLs, DSPs, microcontroladores, generadores de funciones, etc.

La transmisión de la energía mecánica puede ser realizada de dos formas diferentes, dando lugar a una nueva clasificación de estos motores. Por un lado, la transmisión puede realizarse de forma directa, es decir, el desplazamiento se obtiene gracias al efecto piezoeléctrico inverso que se aplica en su totalidad y directamente a la carga. Normalmente esta transmisión se realiza en los motores de excitación forzada por lo que los movimientos que se obtienen son muy bajos, en torno a los nm o unos pocos $\mu \mathrm{m}$. El otro tipo de transmisión se realiza 
por fricción, y principalmente se utiliza en los motores de excitación resonante. La vibración microscópica se convierte en un movimiento elíptico en el punto de contacto, transmitiéndose el movimiento por fricción. Los desplazamientos son más elevados que en el caso de la transmisión directa.

El interés por utilizar este tipo de actuadores no es nuevo. El primer intento por utilizar este tipo de actuador fue en relojes (Bulova Watch Co. Ltd. 1961) [1] para mover los engranajes mediante la vibración de una horquilla en contacto con los engranajes. En años posteriores se fueron desarrollando distintos motores ultrasónicos, H. V. Barth, [2], V. V. Lavrinenko, [3], o Vasiliev, [4], pero no obtuvieron ningún resultado práctico. Hubo que esperar hasta el año 1980, en el que T. Sashida, [5], consiguió satisfacer las condiciones necesarias para un uso práctico de un motor ultrasónico, sin embargo la superficie de contacto entre el rotor y las piezas vibratorias sufrían un gran desgaste y se rasgaban con gran facilidad. Por ello, en 1982 creó un nuevo motor con un principio de funcionamiento diferente a los anteriores, el motor ultrasónico de onda viajera, [6].

\section{MOTORES RESONANTES}

Para situarlos en las clasificaciones que anteriormente fueron mencionadas, se puede decir que los motores ultrasónicos son actuadores piezoeléctricos excitados de forma resonante y que transmiten el par por fricción entre el estator y el rotor.

El principio de funcionamiento de los motores ultrasónicos es el de crear un movimiento elíptico en el punto de contacto entre rotor y estator que dé lugar al movimiento del motor. La creación de este movi- miento elíptico puede realizarse de diferentes maneras. Principalmente se utilizan dos ejes perpendiculares y la composición de sus movimientos armónicos. La forma en la que se generen y se combinen estos movimientos armónicos da lugar a diferentes motores resonantes. Una posible clasificación de los motores piezoeléctricos resonantes se debe al número de modos de vibración. En el caso de un solo modo de vibración se tienen motores de onda estacionaria o motores de onda viajera.

Uno de los métodos para conseguir el movimiento elíptico es la utilización de dos motores resonantes lineales dispuestos perpendicularmente. De esta forma, el punto de contacto recibe el movimiento de los actuadores en dos direcciones perpendiculares, que en conjunto, utilizando un desfase adecuado en las señales de excitación (según las figuras de Lissajous), da lugar al movimiento elíptico deseado. Este tipo de configuración es utilizado para la construcción de motores lineales, situando en la parte superior un elemento deslizante y construyendo plano el punto de contacto entre las cerámicas y el deslizador. En la figura 1 se muestra un análisis por elementos finitos realizado con el software ATILA en el que se puede apreciar el movimiento elíptico en el punto superior. La parte discontínua corresponde a la pieza en reposo.

Otra de las formas de realizar el movimiento elíptico en el punto de contacto es a través de la generación de una onda viajera. Esta modalidad se basa en el hecho de que cuando una onda viajera se propaga a través de un cuerpo elástico finito, las partículas de la superficie realizan un movimiento elíptico. La onda viajera puede ser obtenida a través de la combinación de dos ondas estacionarias desfasadas $\lambda / 4$. A su vez, estas ondas estacionarias se obtienen mediante la excitación de una cerámica polarizada con patrones seno y coseno con la frecuencia correspondiente a la resonancia escogida.
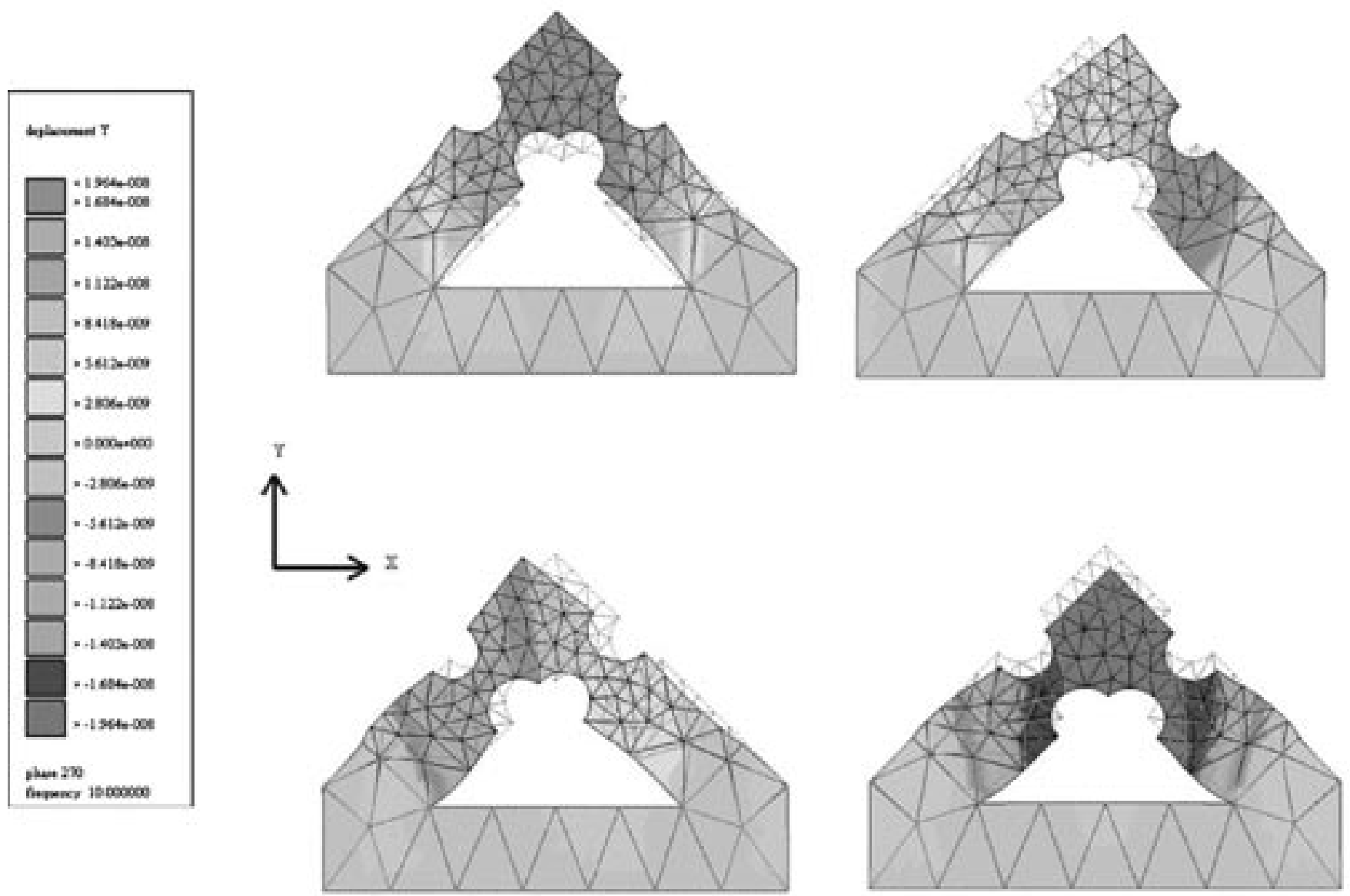

Figura 1. Sucesión de movimientos de un motor piezoeléctrico lineal (formado por dos resonadores longitudinales) en el que se aprecia la trayectoria elíptica del punto de contacto. 
Siguiendo esta aproximación, se pueden fabricar tanto motores giratorios (figura 2) como motores lineales. En el caso del motor lineal, el problema principal radica en la no continuidad del medio en el que se propaga la onda lo que provoca un rebote al final del material y el establecimiento de ondas estacionarias.

La teoría básica de los motores piezoeléctricos de onda viajera puede describirse a partir de la figura 3, [7]. El lugar que describen los puntos ubicados en la superficie superior de los dientes del estator se puede obtener aproximadamente asumiendo primero, que se ha conseguido provocar una onda viajera a lo largo del plano neutro (el plano donde las deformaciones por extensión son cero) y segundo, que el disco anular sobre el que descansan los dientes es muy delgado (i.e. $\mathrm{D} / \mathrm{h}>20)$, por lo que los planos diametrales permanecen perpendiculares al plano neutro después de que este último ha sido deformado (asunción de Kirchhoff). Para describir la superficie del plano neutro deformado se utilizará el vector paramétrico $c(r, \theta, t)$ que describe un punto genérico del plano neutro, situando el sistema de coordenadas en la intersección del plano neutro con el eje axial del motor:

$$
c=\left[r+A_{R}(r) \cos (m \theta-\omega t)\right] e_{r}+\theta e_{\theta}+A_{Z}(r) \cos (m \theta-\omega t) e_{z}
$$

en donde, $A_{R}(\mathrm{r})$ y $A_{z}(\mathrm{r})$ son las amplitudes de la vibración en la dirección radial y axial, respectivamente. Lo siguiente es obtener el vector unitario normal a esta superficie, $n$, sobre el cual se ubica el diente. Para ello calculamos primero las tangentes a la superficie según la dirección radial y circunferencial:

$$
\begin{gathered}
t_{r}=\frac{\partial c}{\partial r}=\left[1+\frac{d A_{R}(r)}{d r} \cos (m \theta-\omega t)\right] e_{r}+\frac{d A_{Z}(r)}{d r} \cos (m \theta-\omega t) e_{z} \\
t_{\theta}=\frac{1}{r} \frac{\partial c}{\partial r}=-\frac{d A_{R}(r)}{d r} m \operatorname{sen}(m \theta-\omega t) e_{r}+\frac{1}{r} e_{\theta}-\frac{A_{Z}(r)}{r} m \operatorname{sen}(m \theta-\omega t) e_{z}
\end{gathered}
$$

Con este resultado obtenemos el vector $n$, mediante el producto interno de ambas tangentes:

$$
n=\frac{t_{r} \times t_{\theta}}{\left|t_{r} \times t_{\theta}\right|}
$$

La mayoría de los motores piezoeléctricos de onda viajera tipo disco se diseñan de tal forma que el desplazamiento del diente sea lo más vertical posible. Esto favorece un mayor contacto y reduce las pérdidas por fricción debidas a desplazamientos radiales adicionales. Conociendo la distancia del plano neutro a la cima del diente, $h_{d}$, el movimiento de esta última se puede representar como:

$$
H(r, \theta, t)=c(r, \theta, t)+h_{d} n
$$

cuyas tres componentes son,

$$
\begin{aligned}
& H_{r}(r, \theta, t)=r_{0}+\frac{A_{R 0}}{r_{0}} \cos (m \theta-\omega t) \\
& H_{\theta}(r, \theta, t)=\theta_{0}+\frac{A_{Z 0}}{r_{0}} \operatorname{sen}(m \theta-\omega t) \\
& H_{z}(r, \theta, t)=h_{d}+\frac{A_{Z 0}}{r_{0}} \cos (m \theta-\omega t)
\end{aligned}
$$

El movimiento descrito por esta última ecuación es el de la elipse centrada en el punto $\left(\theta_{0}, h_{d}\right)$ (figura 4$)$. Claramente cuanto más alto es el diente más grande es la longitud del eje mayor de la elipse. Además, resulta que la elipse es significativamente plana ya que la relación entre la longitud del eje mayor y la del menor es $\mathrm{mh}_{\mathrm{d}}$.

En la figura 5 se muestra el movimiento elíptico descrito por el diente con el avance de la onda viajera. Se observa, como el diente en la parte superior de su movimiento elíptico se desplaza en sentido contrario al del avance de la onda viajera. Por tanto el rotor, que contacta directamente con el diente, gira también en sentido contrario al

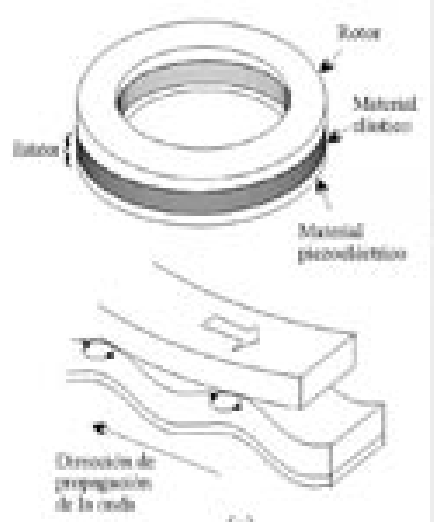

(a)

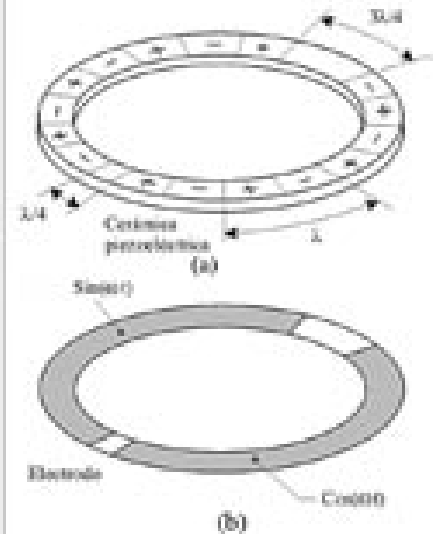

(b)
Figura 2. Esquema de funcionamiento de un motor ultrasónico de onda viajera en motores giratorios y esquema de la cerámica y electrodo necesario para la generación de la onda viajera.

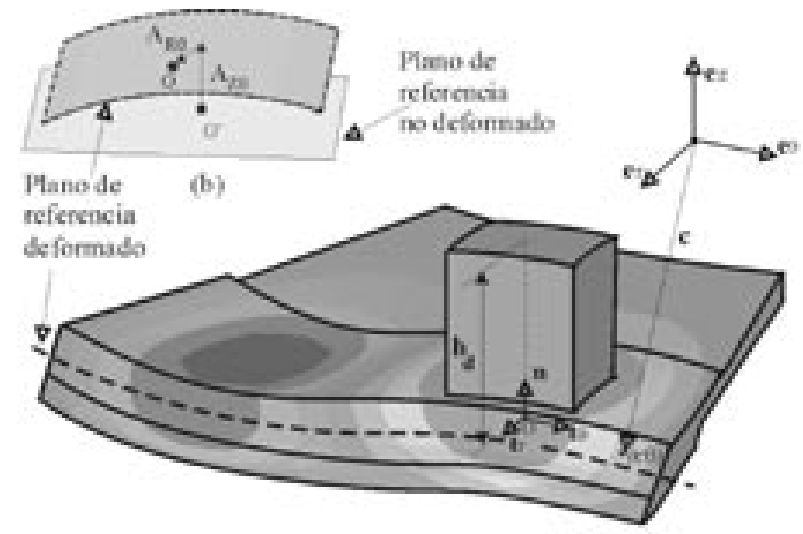

(a)

Figura 3. Flexión resultante de la deformación transversal en la cerámica

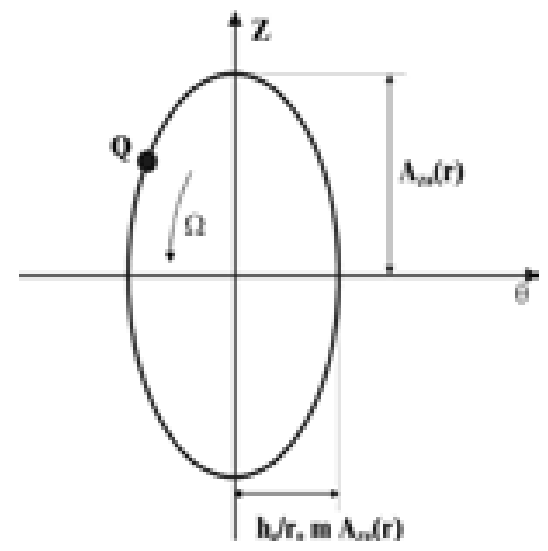

Figura 4. Proyección

de la onda. En la figura 6 se presenta el análisis por elementos finitos de un diseño realizado con entorno ATILA, en el que se aprecia la onda viajera en el estator de un motor ultrasónico excitado.

A partir de las ecuaciones anteriores se puede aproximar la velocidad de rotación del motor. Si asumimos que en estado estable la velocidad tangencial del rotor es igual a la amplitud de la velocidad circunferencial de los puntos en la cima del diente, la magnitud de esta velocidad circunferencial sería:

$$
v=\frac{d\left(H \cdot e_{\theta}\right)}{d t}=m h_{d} \omega \frac{A_{Z 0}}{r_{0}} \cos (m \theta-\omega t)
$$



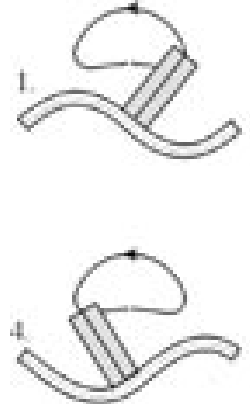

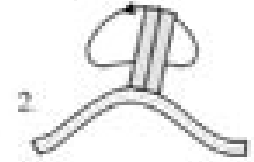

$\square$

Dereccice de peropragacion de la onda

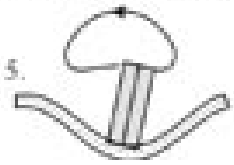

Figura 5. Movimiento elíptico resultante

A partir de esta ecuación y substituyendo la relación entre el número de diámetros nodales y la longitud de onda de la onda viajera $\left(m=2 \pi r_{0} / \lambda\right)$, obtenemos la velocidad de rotación:

$$
\Omega_{\text {rotor }}=\frac{2 \pi}{\lambda} h_{d} \omega \frac{A_{Z 0}}{r_{0}}
$$

En esta expresión queda de manifiesto que la velocidad de rotación $\Omega$ depende inversamente de la longitud de onda de la excitación $\lambda$, y depende directamente de la amplitud de la vibración $A_{\mathrm{zo}}$, de la altura del diente medida desde el plano neutro $h_{d}$ y de la frecuencia de la excitación $\omega$. Esta última en realidad no sólo afecta directamente a $\Omega$, sino también indirectamente ya que la amplitud $A_{z 0}$ depende también de la frecuencia de excitación.

Dentro de la categoría de los motores de tipo disco, hay multitud de posibles modos de excitación (flexión, circunferencial, radial, flexión no-axisimétrica...) para el establecimiento de la elipse en el punto de contacto. Un ejemplo de esto último lo tenemos en el caso de los motores por conversión de modo. En este motor, se hace funcionar al actuador sólo en modo longitudinal, convirtiéndose este movimiento en transversal al atacar el rotor al estator con una pequeña inclinación (unos $6^{\circ}$ ). El gran problema que tienen este tipo de motores es el gran desgaste que se produce en la punto de aplicación (figura 7).

La motivación del continuo estudio de estos motores se debe principalmente a su régimen de actuación en comparación con los motores de DC electromagnéticos. Los motores ultrasónicos pueden producir elevado par (entre 0.1 y $2 \mathrm{Nm}$ ) a velocidades bajas (100-700 rpm). Como consecuencia, pueden suprimirse las etapas de transmisión mejorando la precisión en el posicionamiento. Además, son autoblocantes en ausencia de excitación, muy silenciosos, presentan ausencia de interferencias (no utilizan campo magnético para su funcionamiento) y tienen una estructura simple y predominantemente plana.

Sin embargo no todo son ventajas ya que es necesario para el buen funcionamiento del motor un material muy resistente entre las piezas de contacto, así como una fuente de potencia eléctrica de alta frecuencia para el control del motor. Además la vida útil y eficiencia de estos motores es más reducida que en los electromagnéticos [8].

En la figura 8 se comparan las regiones de operación típicas de los motores electromagnéticos DC con escobillas y de los piezoeléctricos para un rango de potencia entre 1.5 y $5 \mathrm{~W}$. En contraste con las altas velocidades y bajos pares de los motores electromagnéticos, los motores piezoeléctricos son capaces de ejercer pares relativamente altos a baja velocidad, sin reducción mecánica alguna. Si a estas características de operación se le suman las otras ventajas detalladas anteriormente, se tiene entonces un actuador especialmente prometedor para aplicaciones en robótica, en protésica y en subsistemas en el sector de la automoción entre otras. De hecho ya se han aplicado estos motores a algunos subsistemas como cámaras fotográficas, a equipos médicos de resonancia magnética y reposa-cabezas en automóviles de gama alta. A continuación se detallan someramente estos campos de aplicación.

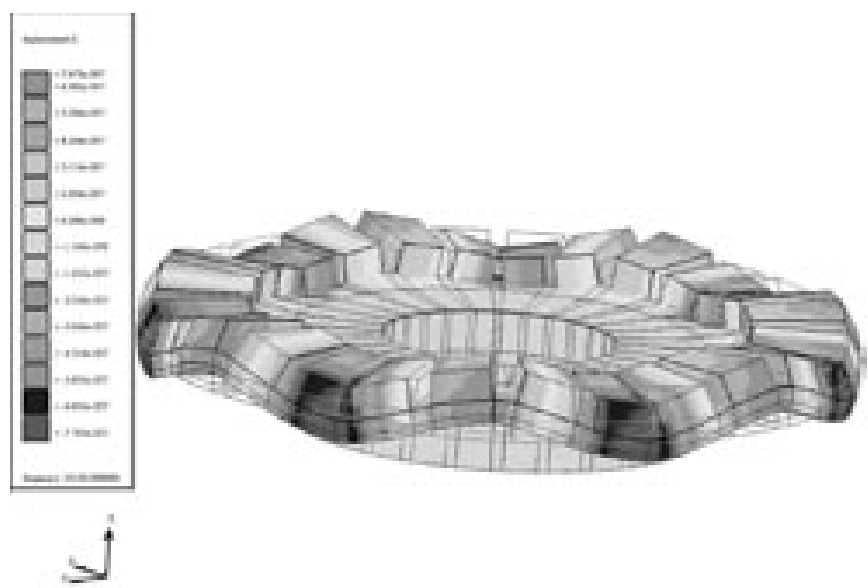

Figura 6. Estator de un motor ultrasónico de onda viajera diseñado con el programa ATILA.

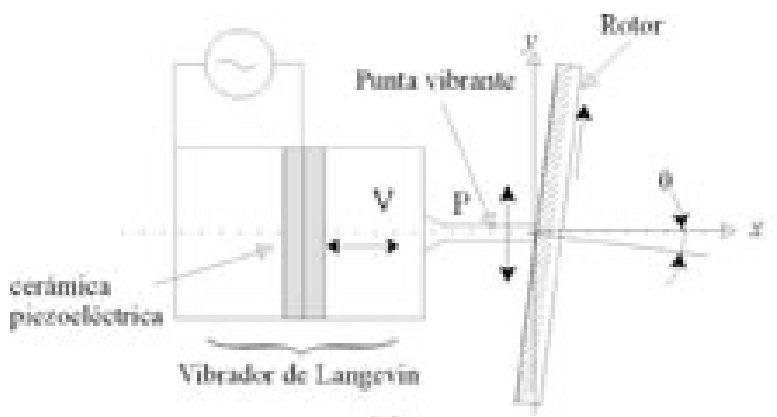

(a)

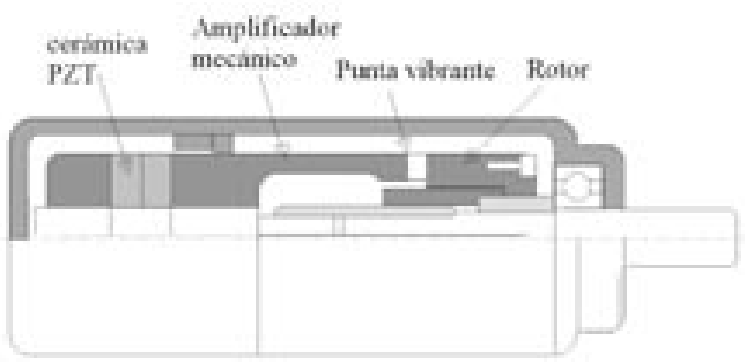

(b)

Figura 7. Actuador piezoeléctrico de conversión de modo.

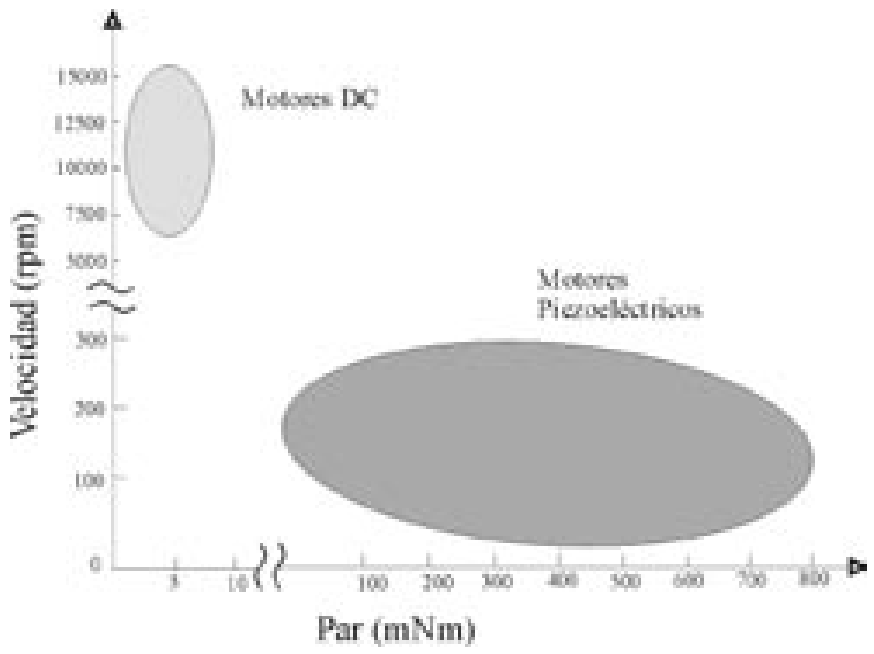

Figura 8. Regiones de operación de motores DC con escobillas y motores piezoeléctricos en el rango de 1.4 a 5 vatios 
Robótica: El potencial que tienen este tipo de actuadores en robótica y protésica ha provocado el interés de muchos investigadores, debido al bajo ruido que desprenden y a la baja inercia que tienen. Schoenwald et al. [9] ya utilizó en 1988 un motor ultrasónico en una mano de un robot, al igual que Ito et al. [10] que usó motores ultrasónicos como actuadores de los tres grados de libertad de una mano protésica, o Pons et al. [11] que utilizaron este tipo de motor para realizar el movimiento de prono-supinación de la muñeca en una mano protésica (figura 9).

Espacio: En el espacio las máquinas deben operar a baja velocidad, para prevenir posibles reacciones debidas a la operación en vacío y la ausencia de gravedad. Por este motivo Das et al. [12] investigaron el efecto de las bajas temperaturas y el vacío en estos motores. Además de las buenas características en cuanto velocidad que ofrecen estos motores se une la falta de necesidad de lubricación, por lo que los hacen muy aptos para su uso en exploraciones interplanetarias.

Automoción: Es probablemente uno de los campos de aplicación que presentan un mayor potencial. Sus características de bajo ruido, no necesidad de engranajes, muy buena relación par-tamaño hacen que estos motores sean idóneos para su uso en el automóvil (reposa cabezas eléctricos, elevalunas, techos solares, etc).

Posicionamiento de precisión: Debido a la ausencia de engranajes, la precisión en el posicionado puede ser muy elevada por lo que son muy apropiados en dispositivos tales como los posicionadores para la fabricación de semiconductores.

Bienes de consumo: Estos motores ya están siendo utilizados en diversos aparatos, como los motores de enfoque automático en algunas cámaras de fotos (figura 10), cortinas automáticas para edificios o impresoras de matrices de puntos.

Entre las numerosas líneas de investigación abiertas, autores como P. Pertsch et al., [13], centran sus estudios en mejorar distintas propiedades de los materiales piezoeléctricos que forman el motor ultrasónico. Otros investigadores, K. Spanner, [14], sin embargo buscan nuevas aplicaciones en las que utilizar toda esta familia de actuadores. Otras líneas de investigación se centran en mejorar los motores actuales o algunos de sus componentes (Nakamura et al. [15]), investigan en nuevas técnicas de modelado para el estator de motores de onda viajera (Pons et al. [16]) e incluso se investiga en nuevos principios de funcionamiento de estos motores (Hu et al. [17]).

\section{ACTUADORES FORZADOS}

Los actuadores multicapa son motores piezoeléctricos de actuación directa que pueden ser excitados tanto de forma resonante como forzada. Los actuadores multicapa están fabricados a partir de distintas placas delgadas de material cerámico piezoeléctrico apiladas de forma que entre dos placas contiguas está convenientemente depositado el electrodo común. El apilamiento de elementos piezoeléctricos es sometido entonces a un tratamiento térmico de forma que se obtiene un dispositivo compacto, de elevada rigidez que permite a la vez tiempos de respuesta reducidos con voltajes de comando relativamente bajos [18].

Cada una de las láminas está polarizada en la dirección de su espesor de forma que el sentido de polarización de láminas adyacentes es opuesto, véase la figura 11. De esta forma, y gracias a la disposición de los electrodos, el dispositivo se comporta eléctricamente en paralelo y mecánicamente en serie obteniéndose como consecuencia un desplazamiento mayor con voltajes reducidos.

Algunas ventajas de los actuadores multicapa frente a su equivalente con una sola capa son que la transmisión de salida es mayor, en un factor igual al número de capas; su impedancia eléctrica es menor

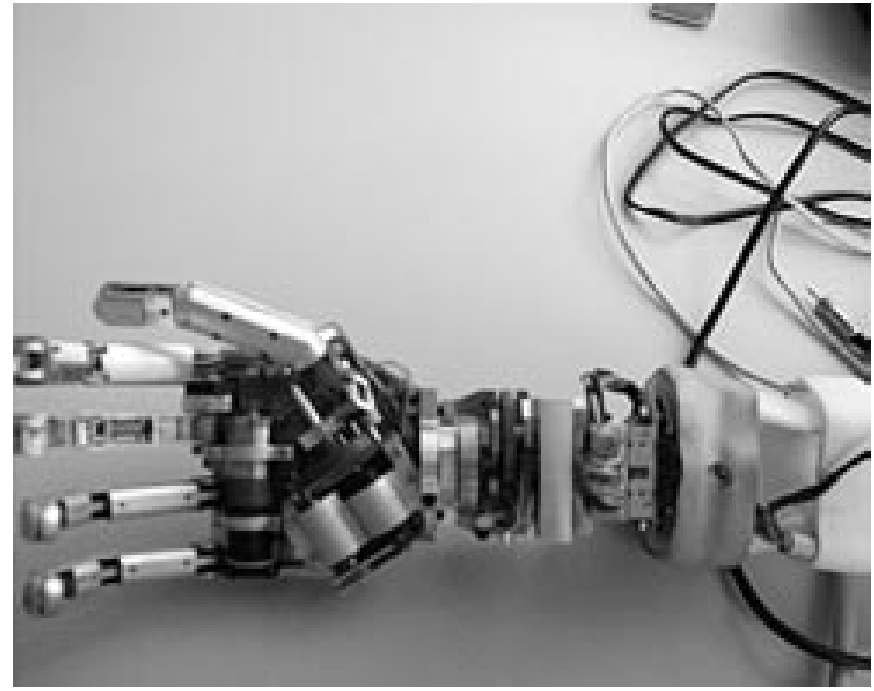

Figura 9. Mano protésica con motor ultrasónico para el movimiento de prono-supinación de la muñeca (proyecto MANUS)

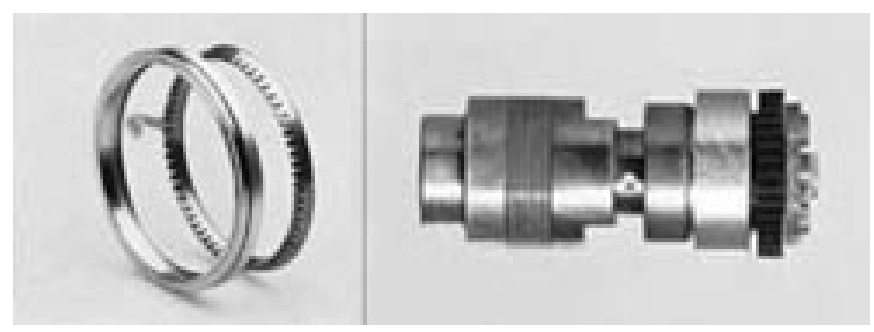

Figura 10. Motor ultrasónico utilizado en la lente de una cámara fotográfica, cortesía de Canon
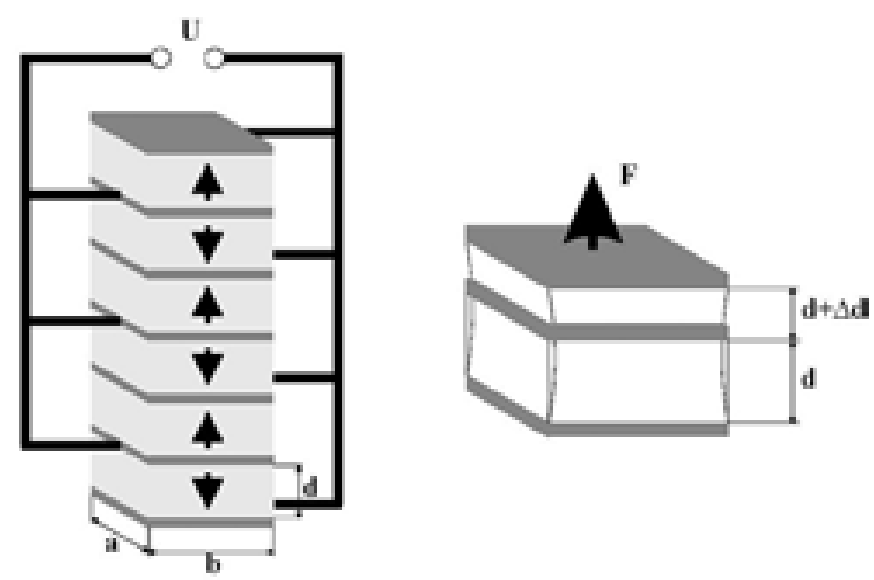

Figura 11. Actuador lineal multicapa y modo de desplazamiento

en un factor de $N^{2}$, siendo $N$ el número de capas, reduciendo de esta manera su propio ruido; la polarización es además mucho más fiable debido a que las láminas son muy finas y además las láminas pueden ser diseñadas de forma individual. Como contrapartida el coste de un multicapa es proporcional al número de capas que contenga, la sensibilidad del voltaje de salida es menor en un factor igual al número de capas y las juntas de pegado incrementan las pérdidas mecánicas, al igual que un desalineamiento de las capas [19]. 
Dos consecuencias importantes se obtienen de estas características: en primer lugar, para obtener niveles de desplazamiento mayores es necesario el uso de multiplicadores mecánicos del desplazamiento que pueden permitir alcanzar niveles de hasta algunas décimas de milímetro. Por otro lado, las precisiones en el posicionamiento pueden ser en algunos casos mayores que un nanómetro por lo que son sistemas principalmente utilizados como micro-posicionadores ultraprecisos (figura 12) o como actuadores en dispositivos de absorción de vibraciones de muy bajo nivel de desplazamiento (control activo). También pueden ser utilizados como fuente de excitación de actuadores resonantes lineales o en equipamiento óptico.

En cuanto a las líneas de investigación sobre este tipo de actuadores, se pueden encontrar líneas dedicadas al estudio del comportamiento de los motores o elementos de ellos. En particular, Rößger, [20], investiga la pérdida de potencia y como afecta este hecho en la máxima frecuencia de operación en actuadores construidos con diferentes materiales o Emanetoglu, [21], analiza la necesidad de controlar y compensar la temperatura para este tipo de estructuras multicapas; otras líneas están basadas en posibles aplicaciones, como Yao et al. [22] que utilizan un actuador multicapa para la fabricación de un micromotor o Versteyhe et al. [23] que desarrollan un completo sistema de posicionamiento XYZ.

Otro tipo de actuador forzado es el lineal cíclico. El esquema de funcionamiento de estos actuadores está fundamentado en la observación del movimiento cíclico de algunos anélidos [18]. Son actuadores capaces de provocar desplazamientos lineales muy elevados, de hasta $250 \mathrm{~mm}$. El actuador lineal cíclico está realmente constituido por tres actuadores piezoeléctricos independientes excitados siempre de forma forzada. Una configuración típica está formada por dos actuadores piezoeléctricos cilíndricos capaces de provocar desplazamientos radiales de contracción y expansión y un tercer actuador cilíndrico lineal según se muestra en la figura 13.

La operación de desplazamiento se obtiene en este tipo de actuador de acuerdo con una secuencia de actuación cíclica tal como muestra la figura 13 que comprende los siguientes pasos:

1. La tensión es aplicada al primer elemento que se contrae quedando firmemente fijado al eje de desplazamiento.

2. El segundo elemento recibe en este momento la tensión de forma que se expande axialmente, produciéndose un desplazamiento equivalente a la mitad de la deformación del segundo elemento.

3. En este instante el tercer elemento es actuado de forma que queda fijado al eje central.

4. Se retira la tensión del primer elemento de forma que este deja de permanecer fijo al eje central.

5. El segundo elemento se contrae desplazando el eje central en el sentido de contracción, en una magnitud de nuevo la mitad de la deformación del segundo

elemento.

6. El ciclo se completa relajando la tensión del tercer actuador.

De la forma indicada en esta secuencia de actuación se pueden obtener velocidades de desplazamiento que oscilan entre $4 \mathrm{~nm} / \mathrm{s}$ y 2 $\mathrm{m} / \mathrm{s}$. La fuerza de actuación puede alcanzar alrededor de $2 \mathrm{~N}$ y se pueden obtener resoluciones en el posicionamiento de unos pocos nanómetros.

En general se puede decir de los actuadores piezoeléctricos pueden utilizarse allí donde sean necesarias resoluciones extremadamente elevadas (micro y nano-posicionadores), incluso mejores que $1 \mathrm{~nm}$. Sin embargo deben ser operados siempre en lazo cerrado debido a su comportamiento no lineal, con histéresis. Dependiendo de la disposición de actuación, su respuesta en frecuencia puede ser extremadamente elevada alcanzando incluso los $10 \mathrm{MHz}$. Las desventajas principales

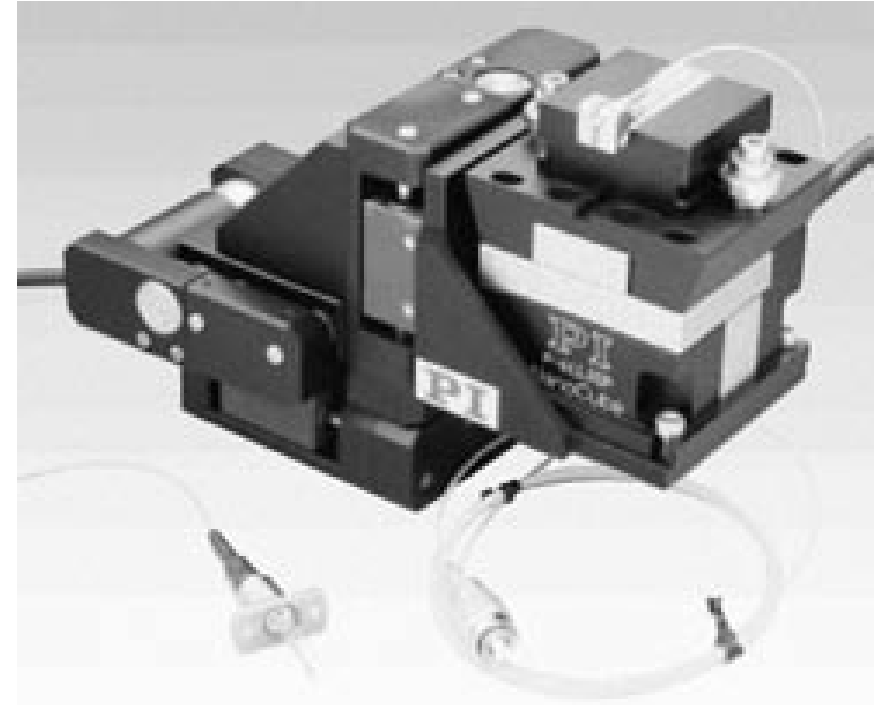

Figura 12. Microposicionador M-110 XYZ de Politec PI

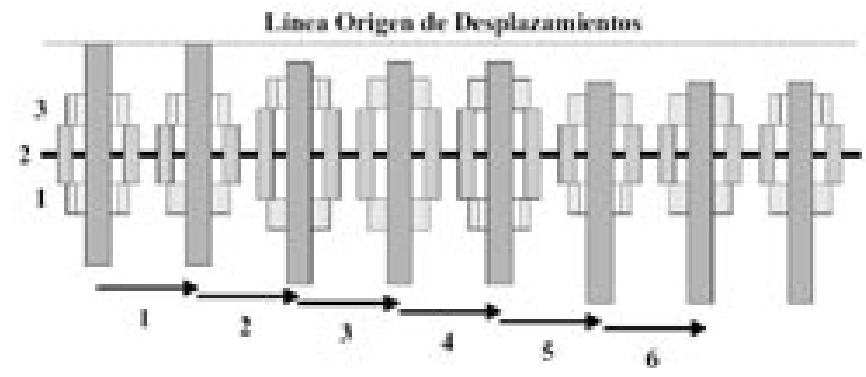

Figura 13. Secuencia de actuación en los actuadores lineales cíclicos.

emanan de los elevados voltajes necesarios para su operación que pueden alcanzar algunos kilovoltios y de los reducidos desplazamientos que han de ser mecánicamente amplificados.

Por último cabe resaltar los vibromotores cuyo principio de funcionamiento es similar al que se usa en cualquier mecanismo general de conversión de movimiento vibratorio en movimiento continuo [24]. Este tipo de motores operan a baja frecuencia, no excediendo por norma de $1 \mathrm{KHz}$. Los vibromotores convierten vibraciones de alta frecuencia (a partir de $20 \mathrm{KHz}$ ) en un movimiento continuo o paso a paso. Por tanto, la diferencia que tienen los vibromotores frente a otros mecanismos que también convierten vibraciones en movimientos rotatorios o lineales es la frecuencia en la que se convierten dichas vibraciones. Otra de las características de los vibromotores es la pequeña amplitud que tienen las vibraciones, en torno a $10^{-2}-10^{-4} \mathrm{~mm}$. En el caso de dispositivos de alta velocidad, las amplitudes pueden llegar a ser de 0.1-0.2 mm. Para hacer funcionar este tipo de motores basta utilizar cualquiera de los mecanismos de excitación utilizados en los motores electrocerámicos.

Este tipo de dispositivo está siendo utilizado en bastantes aplicaciones como por ejemplo en dispositivos de movimiento continuo tanto lineales como rotatorios, con un amplio rango de control de velocidad y pequeñas dimensiones; como posicionadores de gran precisión [25], que poseen una gran sensibilidad y baja constante de tiempo, obteniendo rápidas respuestas en la parada del posicionador; como dispositivos de memoria en discos magnéticos por su pequeño tamaño; como ejecutores de leyes de movimiento, como vibraciones periódicas, etc; gracias a su pequeño tamaño y su gran resolución son utilizados en los scanners y como drivers para micromanipuladores.

Las líneas de investigación sobre los vibromotores se centran principalmente en la búsqueda de aplicaciones, sobre todo en microposicionadores (Saitou et al. [26], Ferreira et al. [27]). 


\section{CONCLUSIONES}

Como se ha expuesto anteriormente, los motores electrocerámicos pueden ser clasificados de diferentes maneras, ya sea por su forma de excitación o por la forma en la que se transmite la energía mecánica del estator al rotor. En función de la aplicación que se requiera, deberá ser usado un tipo especial de actuador. Aplicaciones que requieran gran precisión, como posicionadores ultraprecisos serán realizadas con motores de transmisión directa, donde se obtienen desplazamientos muy bajos a cambio de una gran precisión. Por el contrario, aplicaciones que no requieran tanta precisión y en las que el par requerido sea mayor contarán con motores resonantes con transmisión de energía por fricción (como los motores ultrasónicos de onda viajera).

Estos últimos actuadores pueden ser tanto lineales como rotativos y son especialmente adecuados para un funcionamiento óptimo en aplicaciones como robótica, protésica o en la industria de la automoción, debido al elevado par a velocidades bajas que pueden desarrollar, ausencia de ruido durante su funcionamiento y su estructura simple y compacta.

Entre los actuadores utilizados para el posicionamiento preciso destacan los multicapa, más caros que los monocapa pero con una transmisión de salida mucho mayor (proporcional al número de capas), los lineales cíclicos, de resoluciones extremadamente elevadas pero que deben ser operados en lazo cerrado por la aparición de la histéresis o los vibromotores, pequeños y de gran resolución.

En definitiva, el campo de aplicación es amplio y las distintas variedades de motores electrocerámicos permiten ventajas comparativas en relación con tecnologías clásicas. En este sentido, el presenta tranajo ha presentado las íneas de investigación abiertas que fundamentalmente deben incidir en la mejora de las características funcionales de los diversos dispositivos.

\section{BILBLIOGRAFÍA}

1. F.J. Britten. "Watch and clock maker's handbook, dictionary and guide". Antique Collectors Club, 109-110 (1978).

2. H.V. Barth. "Ultrasonic driven motor". IBM Technical Disclosure Bulletin 16 (1973).

3. V.V. Lavrinenko, V.S. Vishnevski, and I.K. Kartashev. "Izvestiya Vysshikh Uchebnykh Zavedenii". Radioelektonica, 13 [57] (1976).

4. P.E. Vasiliev. UK Patent Application GB 2020857 A. (1979).

5. T. Sashida. Oyo Butsuri, 51, 713-720 (1982).

6. T. Sashida. Japanese Patent Disclosure 58-148682 (1983).

7. H. Rodríguez. "Modelado, diseño y control de motores piezoeléctricos de onda viajera: su aplicación a manos protésicas y robóticas", Tesis doctoral, Universidad Politécnica de Madrid. (2001).

8. S. Ueha, Y. Tomikawa, M. Kurosawa, N. Nakamura. "Ultrasonic Motors", Clarendon Press, Oxford (Reino Unido) 1993.
9. J.S. Schoenwald, P.M. Beckham, R.A. Rattner, B. Banderlip, B.E. Shi. “Exploiting solid state ultrasonic motors for robotics". Proc. IEEE 1988 Ultrasonic Symp., 1 513-517 (1988).

10. K. Ito, H. Nagaoka, T. Tsuji, A. Kato, M. Ito. "An EMG controlled prosthetic forearm with three degrees of freedom using ultrasonic motors". Transaction of the Japanese Society of Instrument and Control Engineers, 27 [11] 12811288 (1991).

11. J.L. Pons, H. Rodríguez, R. Ceres. “High mobility hand prosthesis: A mechatronic approach". Proc. Of the $2^{\text {nd }}$ International Conf. On Recent Advances in Mechatronics, 1 473-477 (1999).

12. H. Das, X. Bao, Y. Bar-Cohen, R. Bonitz, R. Lindemann, M. Maimone, I. Nesnas, C. Voorhees. "Robot manipulator technologies for planetary exploration". Proc. Of the $6^{\text {th }}$ annual International Symposium on Smart Structures and Materials, [1] 3668-3717(1999).

13. P. Pertsch, H. Marth, P. Anger, A. Feltz. "Low-Temperature Properties of Piezoelectric Actuators". Proc. Of the $7^{\text {th }}$ International Conference on New Actuiators \& International Exhibition on Smart Actuators and Drive Systems, 41 (2000).

14. K. Spanner. "Breakthrough in piezo actuator applications". Proc. Of the $7^{\text {th }}$ International Conference on New Actuators \& International Exhibition on Smart Actuators and Drive Systems, 236 (2000).

15. K. Nakamura, J. Satonobu, D. Lee, S. Ueha. “An Optimum Design for the Hybrid Transducer Ultrasonic Motor in Symmetrical Structure". IEEE Ultrasonic Symposium, 703 (1998).

16. J.L. Pons, H. Rodriguez, R. Ceres, L. Calderón. “Novel Modeling Technique for the Stator of Traveling Wave Ultrasonic Motors". IEEE Trans. on Ultras., Ferr. An Freq. Control, en prensa (2003).

17. J. Hu, K. Nakamura, S. Ueha. "Characteristics of a Noncontact Ultrasonic Motor Using Acoustic Levitatio". IEEE Ultrasonic Symposium, 373 (1996).

18. J.L. Pons. "Nuevas Tecnologías para Actuadores en Automatización", XVII Curso de Automática en la Industria, 39 (1997).

19. A. Cochran, G. Hayward. "Multilayer piezocomposite ultrasonic transducers operating below 50KHz". IEEE Ultrasonic Symposium. 953 (1999).

20. D. Rößger, E. Hennig. "Investigations of the Loss Power of Stacked Actuators". Proc. of the $7^{\text {th }}$ International Conference on New Actuators \& International Exhibition on Smart Actuators and Drive Systems, 415 (2000).

21. N.W. Emanetoglu, G. Patounakis, S. Muthukumar, Y. Lu. "Analysis of Temperature Compensated SAW Modes in $\mathrm{ZnO} / \mathrm{SiO} 2 / \mathrm{Si}$ Multilayer Structures". IEEE Ultrasonics Symposium (2000).

22. K. Yao, B. Koc, K. Uchino. “Longitudinal-Bending Mode Micromotor Using Multilayer Piezoelectric Actuatos". IEEE Transactions on Ultrasonics, Ferroelectrics and Frecuency Control, 48 [4] 1066 (2001).

23. M. Versteyhe, D. Reynaerts, F. Al-Bender, H. Van Brussel. "A Rigid and Accurate XYC Positioning System Based on a Piezo-Electric Stepper Drive". Proc. of the $7^{\text {th }}$ International Conference on New Actuators \& International Exhibition on Smart Actuators and Drive Systems, 300 (2000).

24. K. Ragulskis, R. Bansevicius, R. Barauskas, G. Kulvietis. Vibromotors for precision microrobots. Hemisphere publishing corporation, New York (USA) 1988.

25. K. Ragulskis, R. Bansevicius, R. Kurylo. "Positioning in plane using high frecuency vibrations". Proc. of IV World Congress on the Theoty of Machines and Mechanisms, 11-12 (1975).

26. K. Saitou, D. Wang, S. Wou. “Externally Resonated Linear Microvibromotor for Microassembly". Journal of Microelectromechanical Systems, 9 [3] (2000).

27. A. Ferreira, P. Minotti, P. Le Moal. “New Multi-degree cf Freedom Piezoelectric Micromotors for Micromanipulator Applications". IEEE Ultrasonic Symposium, 417 (1995). 